(C) 2020, The Authors. Published by Elsevier Inc. and Fass Inc. on behalf of the American Dairy Science Association ${ }^{\circledR}$. This is an open access article under the CC BY-NC-ND license (http://creativecommons.org/licenses/by-nc-nd/4.0/).

\title{
Longitudinal effects of enrofloxacin or tulathromycin use in preweaned calves at high risk of bovine respiratory disease on the shedding of antimicrobial-resistant fecal Escherichia coli
}

\author{
R. V. Pereira, ${ }^{1 *} \oplus$ C. Altier, ${ }^{2} \oplus$ J. D. Siler, ${ }^{2}$ S. Mann, ${ }^{2} \oplus$ D. Jordan, ${ }^{3} \oplus$ and L. D. Warnick ${ }^{2} \odot$ \\ ${ }^{1}$ Department of Population Health and Reproduction, College of Veterinary Medicine, University of California Davis, Davis 95616 \\ ${ }^{2}$ Department of Population Medicine and Diagnostic Sciences, College of Veterinary Medicine, Cornell University, Ithaca, NY 14850 \\ ${ }^{3} \mathrm{New}$ South Wales Department of Primary Industries, Wollongbar, NSW, Australia 2477
}

\section{ABSTRACT}

The objective of this study was to longitudinally quantify Escherichia coli resistant to ciprofloxacin and ceftriaxone in calves treated with enrofloxacin or tulathromycin for the control of bovine respiratory disease (BRD). Dairy calves 2 to 3 wk of age not presenting clinical signs of pneumonia and at high risk of developing BRD were randomly enrolled in 1 of 3 groups receiving the following treatments: (1) single label dose of enrofloxacin (ENR); (2) single label dose of tulathromycin (TUL); or (3) no antimicrobial treatment (control, CTL). Fecal samples were collected immediately before administration of treatment and at d 2, 4, 7, 14, 21, 28, 56, and $112 \mathrm{~d}$ after beginning treatment. Samples were used for qualification of $E$. coli using a selective hydrophobic grid membrane filter (HGMF) master grid. The ENR group had a significantly higher proportion of $E$. coli resistant to ciprofloxacin compared with CTL and TUL at time points 2,4 , and 7 . At time point 28, a significantly higher proportion of $E$. coli resistant to ciprofloxacin was observed only compared with CTL. The TUL group had a significantly higher proportion of E. coli resistant to ciprofloxacin compared with CTL at time points 2, 4, and 7 . None of the treatment groups resulted in a significantly higher proportion of $E$. coli isolates resistant to ceftriaxone. Our study identified that treatment of calves at high risk of developing BRB with either enrofloxacin or tulathromycin resulted in a consistently higher proportion of ciprofloxacin-resistant E. coli in fecal samples.

Key words: fluoroquinolones, bovine respiratory disease, antimicrobial resistance, enrofloxacin

Received November 30, 2019.

Accepted May 13, 2020.

*Corresponding author: rvpereira@ucdavis.edu

\section{INTRODUCTION}

The use of antimicrobial drugs in food animals, specifically drugs in classes also used in human medicine, remains a contentious public health issue. The quinolone class, used for the treatment of many serious infections in humans, has been regarded as critically important by the World Health Organization (Aidara-Kane, 2012). The majority of quinolones in clinical use belong to the subset fluoroquinolones, which have an added fluorine atom at position 6 of the quinolone molecule that significantly improves its ability to kill bacteria (Andersson and MacGowan, 2003). In the United States, the fluoroquinolones enrofloxacin and tulathromycin are approved for use in nonlactating dairy cattle under 20 mo of age for the treatment or control of bovine respiratory disease (BRD) associated with Mannheimia haemolytica, Pasteurella multocida, Histophilus somni, and Mycoplasma bovis (FDA, 2005, 2008a).

Metaphylaxis is commonly defined as the treatment of an entire group of cattle with an antimicrobial drug with the intent of controlling the incidence of acute onset disease in animals at high risk of developing $\mathrm{BRD}$, although the exact definition for metaphylaxis is still controversial (Ives and Richeson, 2015). Challenges linked to early and accurate diagnosis of BRD in cattle have an influence on the decision to use antibiotics for BRD metaphylaxis (Ives and Richeson, 2015). A recent systematic review and meta-analysis investigating randomized clinical trials indicated a moderate risk reduction in BRD morbidity when using metaphylaxis, with high variability in results observed in different studies (Baptiste and Kyvsgaard, 2017). Some of the main drivers identified included antimicrobial drug class used, definition of metaphylaxis (e.g., cutoff for percent of BRD morbidity compared with presence of specific clinical signs), BRD attack rates, and duration of randomized controlled clinical trials.

Drugs belonging to the macrolide, tetracycline, fluoroquinolone, and phenicol antibiotic classes are 
the most commonly used for metaphylaxis in cattle. A meta-analysis evaluating antibiotic treatments for BRD including 12 antimicrobial drug formulations registered for use in the United States from 1972 to 2013 observed no significant difference in efficacy when comparing enrofloxacin to tulathromycin for treating cattle with BRD (O'Connor et al., 2016). A clinical trial evaluating the effect of metaphylactic treatment of stocker calves with either enrofloxacin or tulathromycin for preventing BRD observed that tulathromycin was superior to enrofloxacin for the control of BRD in high-risk stock cattle. In that study significantly fewer calves that received tulathromycin on arrival required additional treatment for BRD during the first $45 \mathrm{~d}$ after arrival compared with animals receiving enrofloxacin $(33.7 \%$ vs. $18.3 \%$, respectively; Crosby et al., 2018). The difference observed between these 2 studies mirrors the challenges for metaphylaxis studies identified in a study by Baptiste and Kyvsgaard (2017), where variability between study design and presentation of disease on the farm can elicit diverging outcomes from this practice.

In a recent study, researchers screened Escherichia coli isolated from dairy cattle fecal and gastrointestinal tract samples received by the Cornell University Animal Health Diagnostic Center (Ithaca, NY) from 2004 to 2011, and observed that resistance to the drug increased from $1.0 \%$ (6 out of 574) in 2004 to $8.1 \%$ (15 of 185) in 2011 (Cummings et al., 2014). Increased resistance to enrofloxacin during that period coincided with the approval of enrofloxacin in 2008 for use in female dairy cattle under 20 mo of age (FDA, 2008b). A cross-sectional study in preweaned calves also indicated that calves with farm history of treatment with enrofloxacin had higher prevalence of shedding $E$. coli resistant to both ciprofloxacin and ceftriaxone (Pereira et al., 2014c). Use of antimicrobials belonging to the same drug class has been shown to result in a selection of resistance to other drugs in the same drug class due to similar or the same resistance mechanism, resulting in phenotypic resistance. This has been observed in antimicrobials in the quinolone class, and therefore antimicrobial use of enrofloxacin could result in resistance to ciprofloxacin, a fluoroquinolone used widely in human medicine (Hopkins et al., 2005). Resistance to fluoroquinolones can limit therapy options for humans with invasive disease due to Salmonella species, Campylobacter species, and multidrug-resistant Shigella species (Collignon et al., 2016). Commensal E. coli are commonly used as sentinels for antimicrobial resistance in Enterobacteriaceae bacteria (Bywater, 2004).

Regardless of the benefits of using antimicrobial agents in food-producing animals, considerable concerns from public health, food safety, and regulatory perspectives arise due to the potential for development of antimicrobial resistance (Oliver et al., 2011). Because of the decrease in the approval of new antimicrobials by the US Food and Drug Administration, concomitant with the rise of antibiotic resistance, sustainable use of currently available antimicrobials is urgently needed (Spellberg et al., 2011). This includes studies that provide data for the estimation of the effects of using antimicrobial drugs for metaphylaxis on selection of antimicrobial-resistant bacteria. This type of information is also relevant when comparing alternative approaches that reduce the use of antimicrobial drugs for disease control but which could result in increased use of antimicrobials for treatment of individual animals presenting clinical signs of disease.

The objective of this study is to longitudinally quantify and characterize antimicrobial susceptibility of $E$. coli from fecal samples of preweaned calves receiving metaphylactic treatment of either enrofloxacin or tulathromycin.

\section{MATERIALS AND METHODS}

\section{Ethics Statement}

Fecal samples were collected from preweaned dairy calves (Bos taurus) not presenting clinical signs of pneumonia and at high risk of developing BRD that were housed on a commercial dairy farm in New York State. The research protocol was reviewed and approved by the Institutional Animal Care and Use Committee of Cornell University (Ithaca, NY; protocol number 2014-0094). The farm owner authorized conducting of the study on the farm. Antimicrobial treatments were purchased from and administered under the guidance of the herd veterinarian, who maintained a valid veterinary-client-patient relationship with the farm and considered the use of these drugs as an approach to reduce the prevalence of BRD in animals at high risk of BRD, consenting to the treatments used in this study.

\section{Study Design, Population, and Enrollment}

This study used a randomized controlled trial, where calves were enrolled in the study from May 2015 to July 2015 on a commercial dairy farm in New York State that milked 1,200 Holstein cows. The farm was chosen for convenience and for its history of using antimicrobials for control of BRD in preweaned calves. As per the FDA new animal drug application (NADA) 141-068 and NADA 141-244, enrofloxacin and tulathromycin, respectively, are approved for use in beef and nonlactating dairy cattle for control of BRD in animals at high risk of developing $\mathrm{BRD}$, and for treatment of BRD (FOI, 2005; FDA, 2012). In NADA 141-068, the 
FDA outlines specific factors that classify a population as being at high risk for $\mathrm{BRD}$, and of those factors the one present on the farm where calves were sampled was exposure to wet or cold weather conditions. Routine calf management was conducted by farm employees and followed calf management and feeding practices similar to those observed in other commercial farms in the region. Briefly, newborn dairy calves were fed 4 quarts of colostrum within the first hour of life. Routine milk feeding thereafter consisted of bottle-fed pooled pasteurized nonsaleable milk twice a day, until weaning at approximately $56 \mathrm{~d}$ of life. Samples collected at $56 \mathrm{~d}$ occurred during the weaning process but before calves were moved out of the hutches. Water was offered ad libitum in buckets. Heifer calves were kept in individual hutches or pens with sawdust bedding during the preweaning period (first 8 wk of life) and then moved to group pens. Health-related events (e.g., otitis, pneumonia, and diarrhea) were treated as found necessary by farm employees, following standard operational procedures for the farm, and recorded in the farm's herd management software (Dairy-Comp 305; Valley Ag Software, Tulare, CA). The research group obtained these health-related and treatment records from the herd management software and directly from the farm's drug use notebook. Calves treated with antimicrobial drugs after enrollment were removed from the study.

Calves 14 to $21 \mathrm{~d}$ of age on the day of the visit were allocated to treatment groups using a block randomization sheet generated in Excel (Microsoft Corp., Redmond, WA) for enrollment, which included a randomization of calves for treatment order. Any calves with a recorded on-farm history of prior antimicrobial treatment were excluded from enrollment. This age group was selected because of the higher risk for development of BRD at this stage of life (Heins, 2014). Preweaned calves without clinical signs of pneumonia were randomly allocated to 1 of 3 study groups: (1) receiving a single subcutaneous dose of enrofloxacin (ENR; $7.5 \mathrm{mg} / \mathrm{kg}$ of BW) in the neck following label directions (Baytril 100, Bayer Corp. Agricultural Division, Shawnee Mission, KS; $100 \mathrm{mg}$ of enrofloxacin $/ \mathrm{mL}$ ); (2) receiving a single label dose of tulathromycin (TUL) $(2.5 \mathrm{mg} / \mathrm{kg}$ of BW) following label directions (Draxxin, Pfizer Animal Health, New York, NY; $100 \mathrm{mg}$ of tulathromycin/mL); or (3) control, no antimicrobial drug treatment (CTL). At every farm sampling visit, a list of calves born in the last 14 to $21 \mathrm{~d}$ was generated and ordered by identification number. Any calf that had previously received antimicrobial treatment was immediately excluded from enrollment. The final sheet was used, together with the randomized list generated in Excel, to allocate calves to each treatment group. Farm employees and owner were blinded to the treatment groups. Researchers collecting data and administering treatment were not blinded to the treatment groups.

\section{Sample Collection and Processing}

Single-use gloves were used to collect fecal samples directly from the rectum of calves, longitudinally, starting immediately before administration of treatment and at $2,4,7,14,21,28,56$, and $112 \mathrm{~d}$ after enrollment. Collected samples were used to culture, enumerate, and test antimicrobial susceptibility of commensal E. coli.

Fecal samples were processed following a previous protocol with a few modifications (Mann et al., 2011). Briefly, $7.5 \mathrm{~g}$ of fecal material from each sample was placed in a sterile 7-oz. Whirl-Pak filter bag (Nasco, Fort Atkinson, WI) and mixed with $20 \mathrm{~mL}$ of sterile $0.9 \%$ saline solution. The sample was mixed in a stomacher for $2 \mathrm{~min}$. The remaining filtrate was placed in a second sterile Whirl-Pak filter bag and processed for another $2 \mathrm{~min}$. From this solution, five 1-mL replicates of the specimen were aseptically transferred from the filter bag into 5 -mL cryovials prepared with $1 \mathrm{~mL}$ of glycerol (Thermo Fisher Scientific, Waltham, MA) and tryptic soy broth (Becton, Dickinson and Co., Franklin Lakes, NJ; $1: 1 \mathrm{vol} / \mathrm{vol}$ ) and placed in a $-80^{\circ} \mathrm{C}$ freezer for storage.

\section{Production of Hydrophobic Grid Membrane Filter Master Grids and Colony Replication}

A master grid was generated for each sample to replicate and enumerate bacterial colonies onto antimicrobial agar plates using the method previously described (Dunlop et al., 1998; Mann et al., 2011). Briefly, one replicate of a frozen sample was thawed in a $30^{\circ} \mathrm{C}$ dry bath. Five-fold serial dilutions were made using $0.1 \%$ peptone water with $1 \%$ Tween 80 (Thermo Fisher Scientific). After vortexing, $1.5 \mathrm{~mL}$ of each diluted sample was filtered through a sterile hydrophobic grid membrane filter (HGMF; ISO-GRID, Neogen Corp., Lansing, MI) using an SF-1 Spreadfilter (Filtaflex Ltd., Almonte, Ontario, Canada). The HGMF was aseptically transferred onto MacConkey agar (Becton, Dickinson and Co.) and incubated at $37^{\circ} \mathrm{C}$ for 18 to $24 \mathrm{~h}$.

A grid with fewer than 200 colonies was chosen, to limit the probability of any colony being derived from more than $1 \mathrm{cfu}$ (Jordan et al., 2005). If no dilution produced a usable master grid, another replicate of the sample was thawed, and additional dilutions were used until the condition was met.

Bacterial colonies on the master grid were copied onto new sterile HGMF grids on a series of 5 different agars using an RP-1 HGMF replicator (Filtaflex Ltd., Almonte). These agars, in sequence of replication, 
were Mueller-Hinton (MH; Becton, Dickinson and Co.) containing ciprofloxacin $(1 \mu \mathrm{g} / \mathrm{mL}$ ciprofloxacin hydrochloride; Alfa Aesar, Haverhill, MA), MH containing ceftriaxone (ceftriaxone sodium salt hemiheptahydrate; Acros Organics, Morris, NJ) at 2 different concentrations ( 1 and $4 \mu \mathrm{g} / \mathrm{mL}$ ), chromogenic agar (CHROMagar E. coli; CHROMagar, Springfield, NJ) to identify E. coli colonies, and $\mathrm{MH}$ with no antimicrobial added, to ensure that all colony-forming units transferred to all agar plates. Replicated plates were incubated at $37^{\circ} \mathrm{C}$ for 18 to $24 \mathrm{~h}$. Concentrations of ciprofloxacin and ceftriaxone used in the media for bacterial quantification were based on Clinical and Laboratory Standards Institute breakpoints for enterobacteriacea (CLSI, 2018).

\section{Quality Control}

Escherichia coli was confirmed by typical blue-colored isolates on chromogenic agar. To ensure quality of the filter system, $2 \mathrm{E}$. coli isolates were chosen as positive and negative controls. These were cultured, processed, and replicated as described for replicate samples from study animals. For the negative control, an American Type Culture Collection E. coli isolate (ATCC 25922) was used and confirmed susceptible to all study antimicrobials using the Sensititer Automated Microbiology System's A80 Panel (TREK Diagnostic Systems Inc., Independence, $\mathrm{OH}$ ). For the positive control, a multidrug-resistant isolate harboring the blaCMY-2 gene, as identified by PCR, was used. For evaluation of quality control of the plates and growth conditions, the isolates used for negative and positive control were streaked onto the side of the antimicrobial plates, outside the grid zone, for at least 2 samples per day.

\section{Imaging and Analysis of Colony Growth}

Following incubation, digital images of the HGMF filters on agar were captured using a Canon Power Shot SX110 IS camera (Tokyo, Japan) using software for remote picture to enhance quality (PSRemote, version 1.6.4; Breeze Systems Ltd., Camberley, UK). To identify each colony grown and its grid cell address (row and column position), a customized software for image analysis was used (HGMFRES; NSW Department of Primary Industries, Orange, Australia) as described previously (Jordan et al., 2005). For isolates not inhibited by the antimicrobial drugs in the agar, another software was used to exclude all colonies on the chromogenic agar not showing the typical $E$. coli color reaction (HGMF Image Dr, NSW Department of Primary Industries).

\section{Antimicrobial Susceptibility Testing Using Kirby- Bauer Disk Diffusion}

Two E. coli isolates from HGMF master grids originating from fecal samples collected on the day of treatment (referred to as 0 henceforth) and at 7, 28, and $56 \mathrm{~d}$ after initial treatment were selected and stored in a $-80^{\circ} \mathrm{C}$ freezer until antimicrobial susceptibility testing could be conducted. One isolate was susceptible to both ciprofloxacin and ceftriaxone based on the antimicrobial plates, and the other was either, in order of preference and based on availability, resistant to both ciprofloxacin and ceftriaxone, resistant to only ciprofloxacin, or resistant to only ceftriaxone. If no isolate met one of these criteria, only 1 isolate was frozen. These isolates were recovered onto tryptic soy agar with $5 \%$ sheep's blood (Northeast Laboratory Services, Waterville, ME). Antibiotic susceptibility testing was performed using a Kirby-Bauer disk diffusion agar assay in accordance with the guidelines published by the Clinical and Laboratory Standards Institute, using a modified (not including all antimicrobials) National Antimicrobial Resistance Monitoring System panel of 12 antimicrobial drugs: amoxicillin-clavulanic acid $(\mathrm{Au})$, ampicillin $(\mathrm{Am})$, cefoxitin (Fox), ceftriaxone (Cro), chloramphenicol (Cho), ciprofloxacin (Cip), nalidixic acid (Na), enrofloxacin (Enro), streptomycin (Str), sulfisoxazole (Sul), tetracycline (Te), and sulfamethoxazole-trimethoprim (Sxt).

\section{Environmental Samples and Salmonella Testing}

Environmental samples (drag swabs of floor and walls of pens or hutches) were collected longitudinally, starting before the administration of antimicrobial treatment and at 7 and $14 \mathrm{~d}$ after treatment. All environmental samples and fecal samples collected on d 0, 2, 7, 14, and 28 were cultured for Salmonella using standard bacteriologic culture methods. Samples were enriched in $10 \mathrm{~mL}$ of tetrathionate broth (Becton, Dickinson and Co.) with iodine supplement and incubated at $42^{\circ} \mathrm{C}$ for 18 to $24 \mathrm{~h}$. After incubation, the enriched samples were streaked onto Brilliant Green agar with novobiocin and Xylose Lysine Tergitol 4 (XLT-4; Northeast Laboratory Services), and incubated at $37^{\circ} \mathrm{C}$ for 18 to $24 \mathrm{~h}$.

\section{Statistical Analysis}

The distribution of age in days at time of enrollment between treatment groups was analyzed using ANOVA in JMP PRO 15 (SAS Institute Inc., Cary, NC). Escherichia coli colony-forming units per gram of feces was calculated by applying the following formula: 
$\mathrm{cfu} / \mathrm{g} \cong n \times\left[\left(10^{d i l_{-} 10}\right)+\left(5^{d i l_{-} 5}\right)+\left(4^{d i l_{-}{ }^{4}}\right)+\left(3^{d i l_{-} 3}\right)\right] \times \frac{20}{7.5} \times \frac{1}{2}$,

where $n$ is the count of isolates on the grid of reference, and dil_ $x$ is the number of respective dilutions used to reach the solution providing approximately 50 to 200 isolates per grid, where $x$ represents the specific number of fold dilutions used for each sample to achieve the number of isolates within the target interval (dil_10 is for 10-fold dilutions, dil_5 is for 5-fold dilutions, and so on).

A repeated measure ANOVA in SAS (SAS Enterprise Guide 7.1, SAS Institute Inc.) was used to analyze the treatment effect on $E$. coli cfu per gram $\left(\log _{10}\right)$, ceftriaxone-resistant $E$. coli cfu per gram $\left(\log _{10}\right)$, and ciprofloxacin-resistant $E$. coli cfu per gram $\left(\log _{10}\right)$ over time. Sampling day $(0,2,4,7,14,21,28,56$, and 112) and treatment group, as well as their interaction, were included in the model as fixed effects. Calf individual identifier was included as a random effect. Pairwise comparison between treatment group and sampling date was conducted using Tukey's multiple comparison test. To assess normality, the model residual values were plotted against the predicted values and were considered acceptable for all models.

Effect of treatment at the group level on E. coli counts was analyzed by calculating the proportion of $E$. coli cfu per milliliter with growth on the applicable antimicrobial plate among those grid cells (ciprofloxacin and ceftriaxone) on both the $\mathrm{MH}$ and chromogenic agar control plates. The proportion of E. coli resistant to ciprofloxacin and the proportion of E. coli resistant to ceftriaxone were evaluated for normality assumption

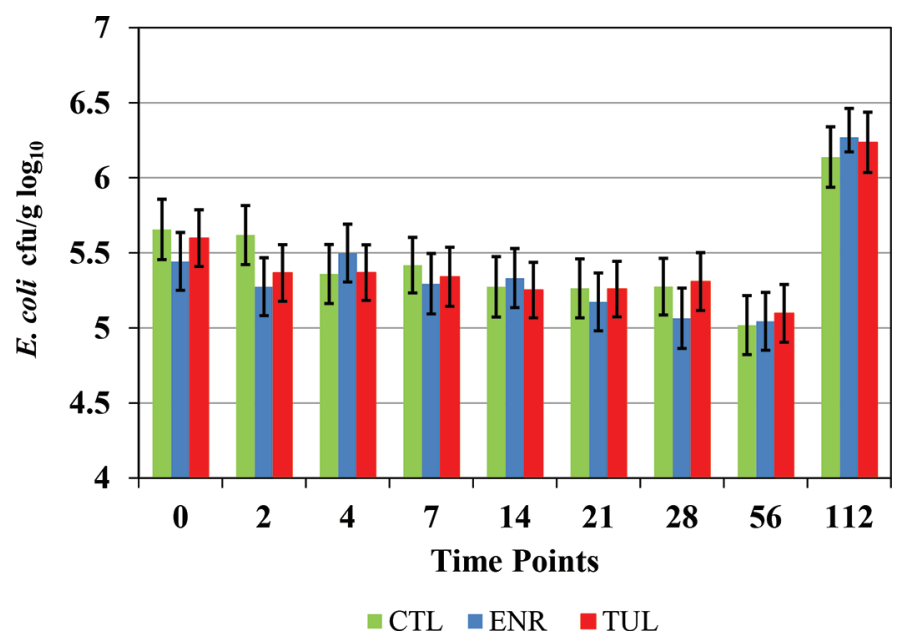

Figure 1. Effect of treatment with enrofloxacin (ENR), tulathromycin (TUL), or no antibiotic (CTL) on the LSM of Escherichia coli cfu per gram $\left(\log _{10}\right)$ counts over time (days relative to treatment). Errors bars represent $95 \%$ CI of the LSM. by visual inspection of the data distribution frequency (histogram) and normal quantile plots, and also by using the Shapiro-Wilk test. The equal variance assumption was evaluated by Levene's and Welch's tests. Because of violation of these assumptions, the nonparametric Kruskal-Wallis test was used to compare the proportion of $E$. coli resistant to ciprofloxacin and the proportion of $E$. coli resistant to ceftriaxone by treatment group, using 1 model for each sampling time point. If a $P$ value below 0.05 was observed using Kruskal-Wallis test, for each sampling time point a Wilcoxon rank sum test model was used to compare each pair of treatment and control groups. To account for multiple individual time point comparisons between treatment groups, a more conservative $P$-value of 0.01 was used to designate a significant difference. Results were displayed using a box-and-whisker plot. Statistical analysis was conducted using the software JMP Pro 15.

\section{RESULTS}

A total of 83 animals were enrolled in the study. From these, 18 calves were excluded due to treatment for diarrhea or loss to follow-up, with 6 being from the ENR group, 7 from the CTL group, and 5 from the TUL group. A total of 65 calves from the initial 83 remained in the study until the last sample was collected on d 112 after enrollment. The mean age in days for calves enrolled in each treatment group was $18.2(95 \%$ CI: $17.2-19.1), 17.5$ (95\% CI: 16.6-18.4), and $18.2(95 \%$ CI: 17.3-19.1) for CTL, ENR, and TUL, respectively $(P=0.45)$.

The least squares means (LSM) for counts of E. coli $\left(\mathrm{cfu} / \mathrm{g} \log _{10}\right)$ over time by treatment group for each time point are shown in Figure 1. No significant differences in LSM counts of $E$. coli were observed between treatment groups for any of the time points. A significant gradual decrease in $\log _{10} E$. coli cfu per gram was observed from time point 0 to 56 (LSM $\pm \mathrm{SE}, 5.5 \pm$ 0.05 to $5.0 \pm 0.05$, respectively), and a significant peak in $\log _{10} E$. coli cfu per gram at time point 112 (LSM \pm SE, $6.2 \pm 0.05 ; P<0.0001)$. The effect of treatment group on LSM counts of $E$. coli $\left(\mathrm{cfu} / \mathrm{g} \log _{10}\right)$ resistant to ceftriaxone over time is illustrated in Figure 2. No significant difference in LSM counts of $E$. coli resistant to ceftriaxone was observed between treatment groups for any of the time points.

The effect of treatment group on LSM counts of $E$. coli $\left(\mathrm{cfu} / \mathrm{g} \log _{10}\right)$ resistant to ciprofloxacin over time is shown in Figure 3. Fecal samples from calves in the ENR treatment group had significantly higher LSM counts of $E$. coli ( $\mathrm{cfu} / \mathrm{g} \log _{10}$ ) resistant to ciprofloxacin compared with CTL and TUL group at time points 2,4 , and 7 . At time point 28 , a significantly higher 


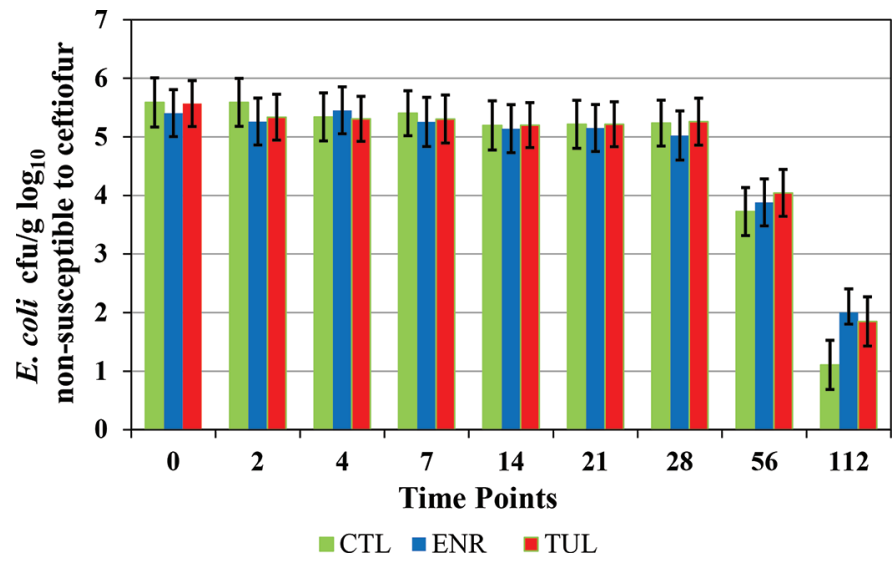

Figure 2. Effect of treatment with enrofloxacin (ENR), tulathromycin (TUL), or no antibiotic (CTL) on the LSM of Escherichia col cfu per gram $\left(\log _{10}\right)$ counts resistant to ceftriaxone over time (days relative to treatment). Errors bars represent $95 \%$ CI of the LSM.

LSM for counts of E. coli (cfu/g $\log _{10}$ ) resistant to ciprofloxacin was observed in the ENR group compared with the CTL group only. The mean cfu per gram of ciprofloxacin-resistant $E$. coli in the ENR, TUL, and CTL treatment groups at time points 2, 4, 7, and 28 are shown in Supplemental Table S1 (https://doi.org/ 10.3168/jds.2019-17989). Calves in the TUL treatment group had significantly higher LSM counts of $E$. coli (cfu/g $\log _{10}$ ) resistant to ciprofloxacin compared with CTL group at time points 2, 4, and 7 .

The effect of treatment group on the proportion of ciprofloxacin-resistant $E$. coli for each time point is illustrated in Figure 4 using box-and-whisker plots. No significant difference was observed between treatment groups for proportion of ciprofloxacin-resistant

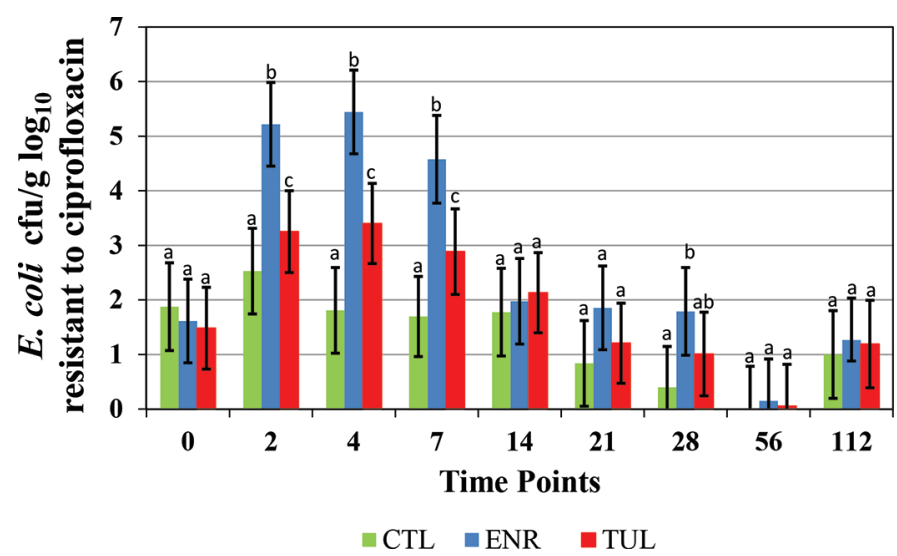

Figure 3. Effect of treatment with enrofloxacin (ENR), tulathromycin (TUL), or no antibiotic (CTL) on the LSM of ciprofloxacinresistant Escherichia coli cfu per gram $\left(\log _{10}\right)$ counts over time (days relative to treatment). Errors bars represent 95\% CI of the LSM. Different lowercase letters $(\mathrm{a}-\mathrm{c})$ indicate treatment groups that had significantly different $(P<0.05)$ LSM.
E. coli at time point 0 . In agreement with the overall mean counts of $E$. coli cfu per gram $\left(\log _{10}\right)$ resistant to ciprofloxacin, a significantly higher proportion of ciprofloxacin-resistant $E$. coli was observed in calves in the ENR treatment group compared with the CTL and TUL groups at time points 2,4 , and 7 . At time point 28, a significantly higher proportion E. coli resistant to ciprofloxacin was observed only compared with the CTL group. Calves in the TUL treatment group had a significantly higher proportion of $E$. coli resistant to ciprofloxacin compared with the CTL group at time points 2,4 , and 7 . The score mean difference values for sampling points where a significant difference was observed is displayed in Table 1.

The effect of treatment group on the proportion of $E$. coli resistant to ceftriaxone for each time point is shown in Figure 5. No significant difference in the proportion of resistant $E$. coli was observed between treatment groups for any of the models comparing proportion of resistant $E$. coli between treatment groups for each sampling time point.

The distribution of the individual E. coli isolates selected for Kirby-Bauer disk diffusion sensitivity testing, according to patterns of susceptibility to ciprofloxacin and ceftriaxone, is shown in Supplemental Table S2 (https://doi.org/10.3168/jds.2019-17989). A heat map with the distribution of the resistance patterns for these individual $E$. coli isolates by treatment group and sampling time point (d) is shown in Figure 6 . For isolates presenting phenotypic resistance to ciprofloxacin $(\mathrm{n}=20)$, only 2 resistance patterns were observed, independent of treatment group, and were AuAmEnroFoxChoCipNaSulTeSxt and AuAmEnroFoxCroChoCipNaSulTeSxt. These 2 resistance patterns differed only by the presence of resistance to ceftriaxone (CRO). The distribution of resistance patterns for these ciprofloxacin-resistant $E$. coli by treatment group and sampling time point (d) is presented in Supplemental Table S3 (https://doi.org/10.3168/jds .2019-17989). No isolate resistant to ciprofloxacin was observed for sampling time point 56. No Salmonella was isolated from any of the environmental samples collected.

\section{DISCUSSION}

Treatment of preweaned calves at high risk of developing BRD with enrofloxacin resulted in a significant increase in the shedding of ciprofloxacin-resistant E. coli in feces for up to 1 wk after administration, compared with calves receiving tulathromycin or not receiving any treatment (Figure 4). Similar results were observed in a previous study using dairy farm records to identify antimicrobial drug treatments in calves to evaluate the 


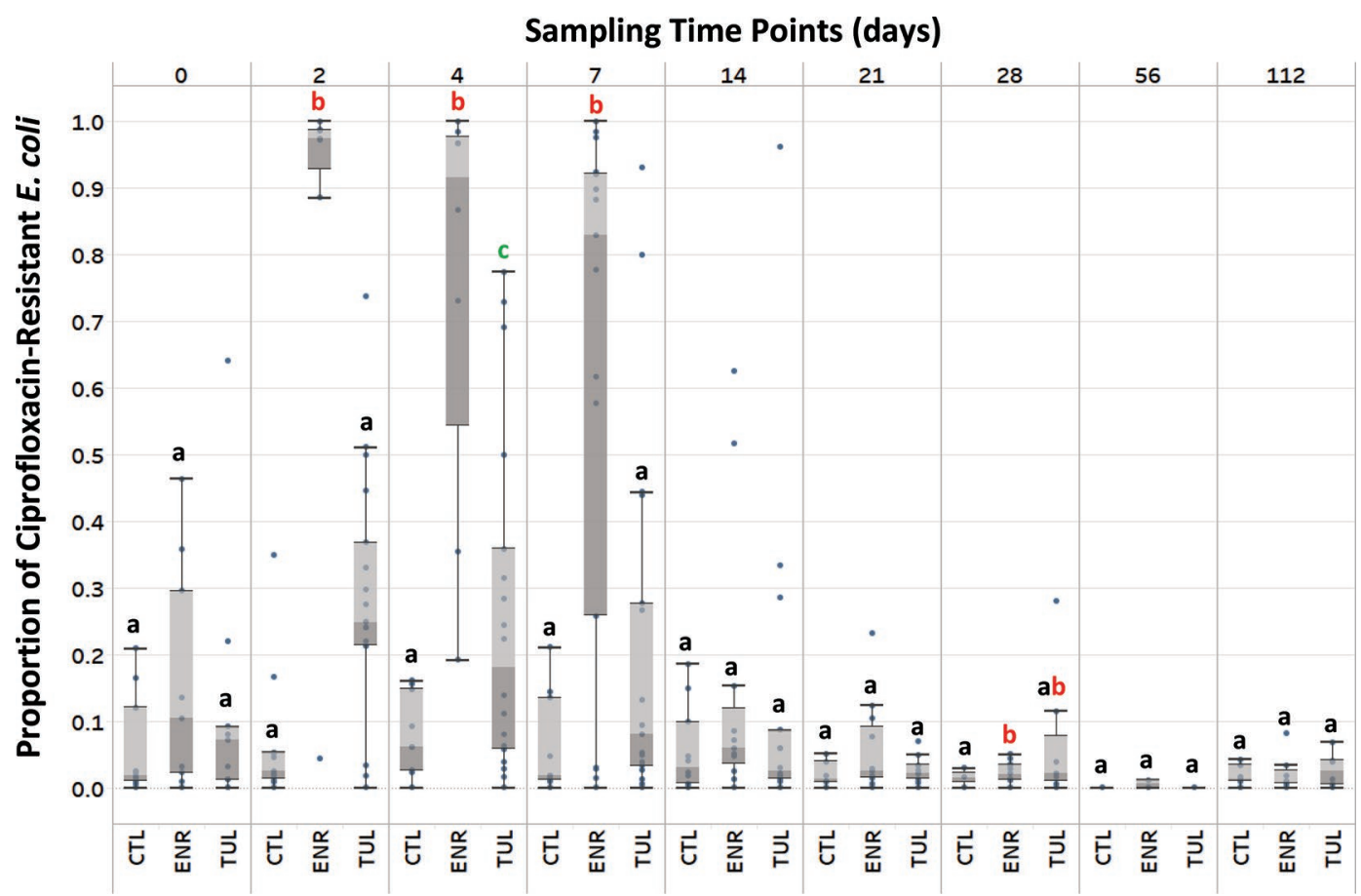

Figure 4. Box-and-whisker plot for treatment with enrofloxacin (ENR), tulathromycin (TUL), or no antibiotic (CTL) on proportion of ciprofloxacin-resistant Escherichia coli over time (days relative to treatment). Data are presented sorted by 75 th percentile, and then by 50th percentile. Each boxplot shows the 50th percentile (median, line within the box), 25th and 75th percentile (box), 10th and 90th percentiles (whiskers), and outliers (dots). Different lowercase letters $(\mathrm{a}-\mathrm{c})$ indicate treatment groups with significant differences in the proportions of ciprofloxacin-resistant $E$. coli, based on Wilcoxon rank sum test comparing treatment groups for each time point $(P<0.01)$.

effect on the prevalence of antimicrobial-resistant Escherichia coli in rectal swabs of preweaned dairy calves (Pereira et al., 2014c). Pereira et al. (2014c) observed that calves treated with enrofloxacin had odds ratios of 2 (95\% CI: 1-4) and 3 (95\% CI: 2-6), respectively, for isolation of fecal $E$. coli nonsusceptible to nalidixic acid and ciprofloxacin, compared with calves not treated with enrofloxacin. One limitation of that study was that treatment event was based on farm records, affecting accuracy of treatment assignment, as well as using only a nonquantitative approach for assigning calf susceptibility status, because susceptibility testing was performed for only 3 fecal E. coli isolates per calf using a Kirby-Bauer disk diffusion assay.

A study by Smith et al. (2017) evaluated the effect of administering metaphylactic treatment with enrofloxacin or no drug treatment in 8-mo-old crossbred beef calves $(\mathrm{n}=288)$ on the prevalence of quinoloneresistant Salmonella and Campylobacter for up to $28 \mathrm{~d}$ after treatment. Their study found no significant effect of metaphylactic treatment with enrofloxacin on the prevalence of Salmonella or Campylobacter resistance to nalidixic acid or ciprofloxacin. Furthermore, their study tested a maximum of one Salmonella or Campylobacter isolate using microbroth dilution for measuring isolates' susceptibility to quinolones, when 1 of these bacteria was cultured from a fecal sample. Their results may have differed from our study findings, due to the use of a different age group. Preweaned calves are known for having a developing enteric microbiota that could facilitate selection and dissemination of antibiotic-resistant bacteria, including those resistant to quinolones (Pereira et al., 2016). Additional differences could be attributed to diet and breed. Smith et al. (2017) also focused on 2 gram-negative bacteria for which shedding incidence in fecal samples may vary. In their study, baseline prevalence of Salmonella was numerically higher in cattle assigned to the pen treated with enrofloxacin compared with the control group, although not statistically significant. Smith et al. (2017) had a mean recovery of Salmonella and Campylobacter from fecal samples of $10.2 \%$ and $12.4 \%$, respectively, and observed no effect of treatment with enrofloxacin on shedding prevalence of either of these bacteria. Our study focused on a quantitative approach evaluating resistance in enteric $E$. coli, which is abundant in the gut microbiota. This could also have resulted in the different outcome for selection of resistance to a fluoroquinolone drug. Escherichia coli has been considered a potential reservoir for resistance genes to other gram- 
Table 1. Results from Wilcoxon rank sum analysis evaluating the difference between the pairs of treatment groups for proportion of ciprofloxacin-resistant Escherichia coli over time ${ }^{1}$

\begin{tabular}{lrrrc}
\hline $\begin{array}{l}\text { Treatment groups } \\
\text { at different time } \\
\text { points }\end{array}$ & & & & \\
\hline Time $=2$ & SMD $^{3}$ & SED $^{4}$ & $Z$-value & $P$-value \\
CTL vs. ENR & -20.80 & 3.71 & -5.59 & $<0.0001^{*}$ \\
TUL vs. CTL & 8.74 & 3.79 & 2.30 & 0.0214 \\
TUL vs. ENR & -21.21 & 3.82 & -5.55 & $<0.0001^{*}$ \\
Time $=4$ & & & & \\
CTL vs. ENR & -21.45 & 3.70 & -5.78 & $<0.0001^{*}$ \\
TUL vs. CTL & 10.22 & 3.79 & 2.69 & $0.0071^{*}$ \\
TUL vs. ENR & -21.64 & 3.88 & -5.56 & $<0.0001^{*}$ \\
Time $=7$ & & & & \\
CTL vs. ENR & -16.94 & 3.71 & -4.55 & $<0.0001^{*}$ \\
TUL vs. CTL & 7.83 & 3.70 & 2.11 & 0.0343 \\
TUL vs. ENR & -14.41 & 3.93 & -3.66 & $0.0003^{*}$ \\
Time $=28$ & & & & \\
CTL vs. ENR & -7.95 & 2.99 & -2.65 & $0.0080^{*}$ \\
TUL vs. CTL & 3.50 & 2.60 & 1.34 & 0.1786 \\
TUL vs. ENR & -4.44 & 3.33 & -1.33 & 0.1825 \\
\hline
\end{tabular}

${ }^{1}$ Data are provided only for sampling time points where $P<0.05$ was observed for at least 1 pair of treatment groups. Difference was considered significant for $P<0.01$.

${ }^{2}$ Time points $=2,4,7$, or $28 \mathrm{~d}$ after treatment. Treatments: CTL $=$ control, no antibiotic; ENR = enrofloxacin; TUL = tulathromycin.

${ }^{3} \mathrm{SMD}=$ score mean difference for Wilcoxon rank sum analysis.

${ }^{4} \mathrm{SED}=\mathrm{SE}$ of the difference.

${ }^{5} P$-value for each pair of comparisons between treatment groups by sampling time points.

$* P<0.01$.

negative enteric bacteria that could uptake antibioticresistance genes originating from $E$. coli (Bailey et al., 2010).

Currently recognized mechanisms of resistance to quinolone drugs include the target-mediated, plasmidmediated, and chromosome-mediated (Aldred et al., 2014). A study using the samples collected during the trial referred to in this study for metagenomics analysis observed no significant difference in relative abundance of genes linked to fluoroquinolone resistance, although a higher overall abundance was observed at d 14 for calves in the ENR group, suggesting that a larger sample size may have been needed to capture potential effects of enrofloxacin on the selection of fluoroquinole resistance in the microbiota as a whole (Foditsch et al., 2019). Although metagenomic approaches generate a massive volume of information about microbiota, an advantage of studies using species-specific quantitative approaches, as used in our study, is the ability to capture differences in microbial resolution level while also securing phenotypic evidence of antimicrobial resistance, which may require a significantly large samples size or sequencing depth when using genome sequencing approaches(Sims et al., 2014). Traditionally, resistance to quinolones has been believed to occur through chromosome-mediated resistance, mainly due to point mutations that result in amino acid substitutions within the topoisomerase subunits GyrA, GyrB, ParC, or ParE, and therefore acquisition of resistance from quinolone-resistant $E$. coli harboring resistance through this mechanism by different gram-negative bacteria is less likely (Hopkins et al., 2005). However, plasmid-mediated quinolone resistance genes that can be transferred between bacteria increase the risk of $E$. coli serving as a reservoir for this resistance mechanism to quinolone drugs for other enteric bacteria. This adds a level of complexity to evaluating the longitudinal effects of treatment with enrofloxacin on dissemination of resistance from commensal enteric bacteria to pathogenic bacteria sharing the same environment (Jacoby et al., 2014). The role of commensal Enterobacteriaceae bacteria as reservoirs of antibiotic resistance genes that could potentially be transferred to pathogenic bacteria has been widely demonstrated (Mesa et al., 2006; Vaidya, 2011).

Another potential route for dissemination of fluoroquinolone resistance shed in the feces is horizontal transmission of resistant bacteria and genes to other animals exposed to feces contaminated with resistant bacteria. In our study calves were housed individually, and, compared with a group-housing scenario, we expect that individually housed calves would exhibit lower dissemination of resistant pathogens shed in the feces. In one experimental trial, researchers evaluated horizontal transmission of fluoroquinolone-resistant $E$. coli between specific pathogen-free pigs that had been inoculated with the resistant bacteria and pigs that were not inoculated (naïve pigs), using a susceptibleinfectious-susceptible model (Andraud et al., 2011). All naïve pigs went from no detectable counts of fluoroquinolone-resistant $E$. coli to greater then $2.0 \times 10^{4} \mathrm{cfu} / \mathrm{g}$ $\log _{10}$ after only $2 \mathrm{~d}$ of contact with inoculated animals. In that study, inoculated pigs had an initial decline in fluoroquinolone-resistant $E$. coli counts in the feces 4 to $5 \mathrm{~d}$ after inoculation, followed by an overall increase from d 6 until the end of the experiment. The authors concluded that inoculated pigs were reinfected by naïve pigs that had started shedding fluoroquinoloneresistant E. coli. In their study, transmission by low shedders (fluoroquinolone-resistant E. coli in the feces between $5 \times 10^{3}$ and $\left.10^{6} \mathrm{cfu} / \mathrm{g}\right)$ was 2.5 times lower than that of high shedders $\left(>10^{6} \mathrm{cfu} / \mathrm{g}\right)$. One similarity with our study is that preweaned calves are considered monogastric animals, as are swine, although this gradually changes to a ruminant gastrointestinal system as calves are weaned and mature (Heinrichs and Lesmeister, 2005). In our study, at time points 2, 4, and 7, calves in ENR treatment not only showed significantly greater shedding compared with TUL and CTL calves but reached shedding levels for ciprofloxacin-resistant E. coli above $10^{5} \mathrm{cfu} / \mathrm{g}$. To our knowledge, no currently 


\section{Sampling Time Points (days)}

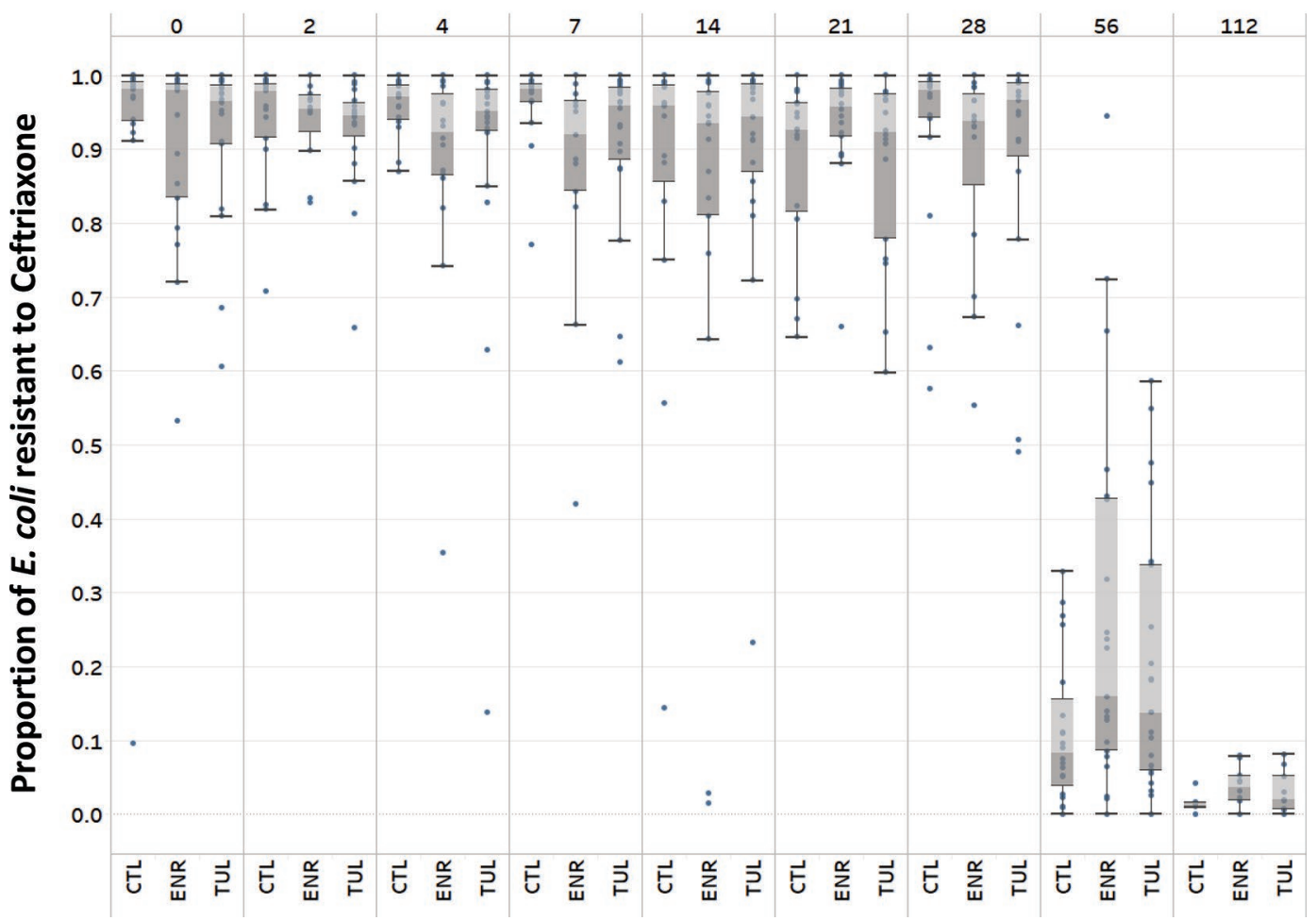

Figure 5. Box-and-whisker plot of the proportion of ceftriaxone-resistant fecal Escherichia coli over time (days relative to treatment) in calves treated once with enrofloxacin (ENR), tulathromycin (TUL), or no antibiotic (CTL). Data are presented sorted by 75 th percentile, and then by 50th percentile. Each boxplot shows the 50th percentile (median, line within the box), 25th and 75th percentile (box), 10th and 90th percentiles (whiskers), and outliers (dots).

published model has estimated the transmission of fluoroquinolone-resistant $E$. coli between preweaned calves; given differences between pig productions systems due to herd management practices, including housing, which could affect transmission rates, extrapolation of results from research conducted in pigs to calves should be carefully acknowledged. Nevertheless, the effects of shedding levels of resistance dissemination and persistence are of great relevance for better understanding of the dissemination of ciprofloxacin resistance in preweaned calves and are an area of relevance for future studies.

Calves treated with a single dose of enrofloxacin at 8 $\mathrm{mg} / \mathrm{kg}$ of BW are expected to have a mean plasma halflife of $7.28 \mathrm{~h}$ (TerHune et al., 2005). Enrofloxacin is a concentration-dependent (rather than time-dependent) bactericidal, and the mean time to maximum enrofloxacin plasma concentration, peak plasma concentration, and area under the concentration versus time curve over a 12-h period have been shown to be $2 \mathrm{~h}, 0.81$ $\mu \mathrm{g} / \mathrm{mL}$, and $7.51 \mathrm{~h}$, respectively. Different pharmacodynamic and pharmacokinetic parameters have been evaluated as potential indicators for reduction of selec- tion of antimicrobial resistance after therapeutic use of an antimicrobial drug. Examples include use of the ratio of peak plasma concentration, or the area under the concentration-versus-time curve over a 24 -h period, to MIC to predict antimicrobial drug efficacy and minimization of selection of antimicrobial-resistant bacteria (Rybak, 2006). However, studies evaluating different dosing regimens of ciprofloxacin (oral route) in a clinical setting have found contradicting results when using these parameters to estimate emergence of antimicrobial-resistant bacteria. As an example, one study evaluating oral ciprofloxacin in humans on selection of $E$. coli in the fecal flora did not observe a significant difference for pharmacodynamic and pharmacokinetic parameter combinations between the subjects in whom resistance emerged and those in whom it did not (Fantin et al., 2009). Results from our study indicate that the selection and shedding of resistance to antimicrobial drugs involve factors that could not be predicted by pharmacodynamics or pharmacokinetics alone, as indicated by a significantly higher proportional shedding of $E$. coli resistant to ciprofloxacin up to $7 \mathrm{~d}$ after a singledose treatment, which exceeds the expected values for 


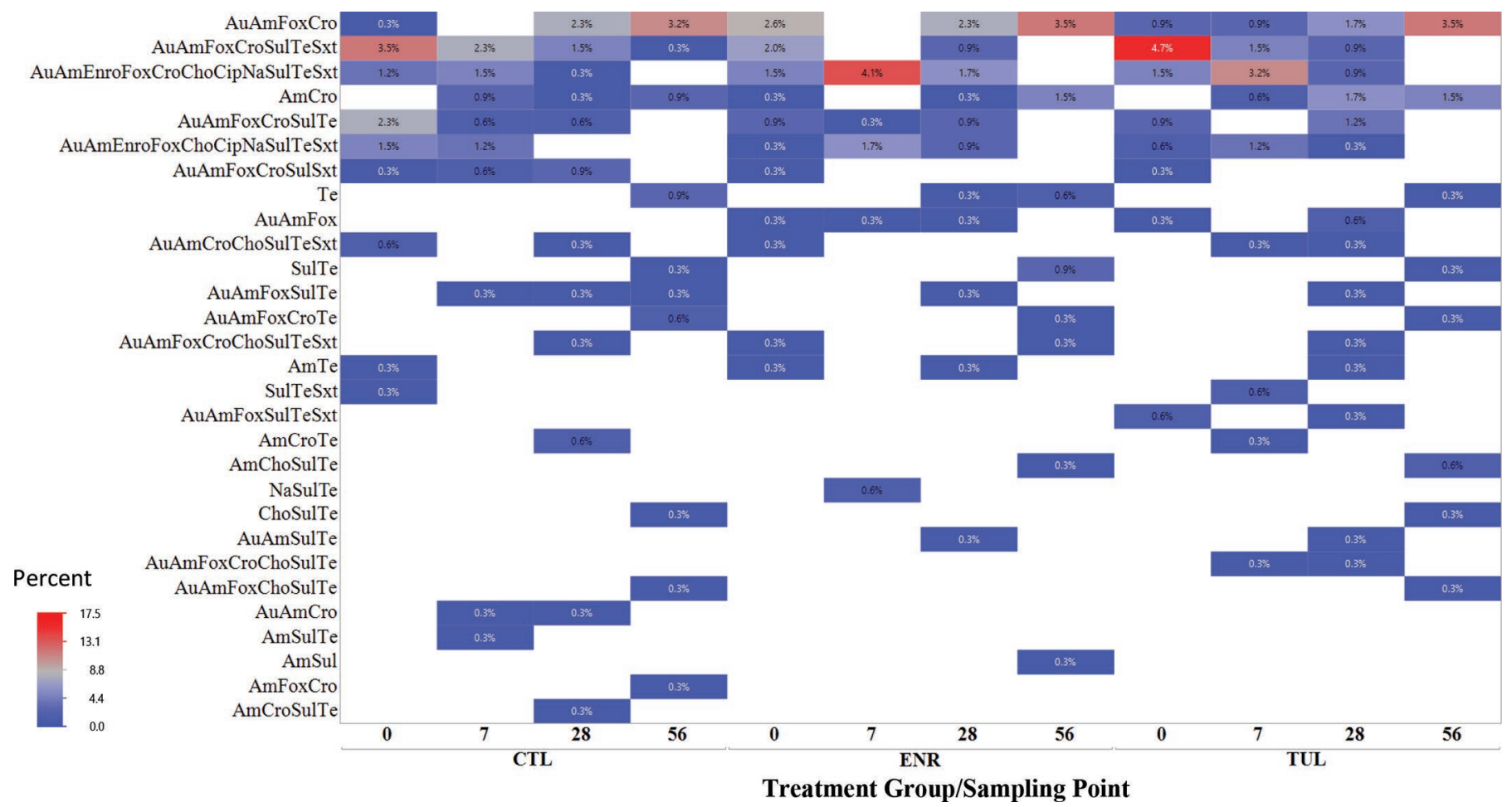

Figure 6. Heat map showing the percent distribution of resistance patterns of all Escherichia coli isolates, as determined by Kirby-Bauer diffusion test, by treatment group $(\mathrm{ENR}=$ enrofloxacin, TUL $=$ tulathromycin, $\mathrm{CTL}=$ control, no antibiotic) and sampling time point $($ days relative to treatment). $\mathrm{Au}=$ amoxicillin/clavulanic acid; $\mathrm{Am}=$ ampicillin; Enro $=$ enrofloxacin; Fox $=$ cefoxitin; Cip $=$ ciprofloxacin; Cro $=$ ceftriaxone; Cho = chloramphenicol; $\mathrm{Na}=$ nalidixic acid; $\mathrm{Str}=$ streptomycin; $\mathrm{Te}=$ tetracycline; $\mathrm{Sxt}=$ trimethoprim/sulfamethoxazole; Sul $=$ sulfisoxazole.

enrofloxacin effect on bacteria based on clinical pharmacologic measures.

An unexpected finding in our study was that, although enrofloxacin has a spectrum of action that includes gram-negative enteric bacteria such as E. coli, we did not observe a significant reduction in the total E. coli cfu per gram in the feces of calves in the ENR group compared with TUL or CTL (Constable, 2004). One study that administered a single dose (12.5 $\mathrm{mg} / \mathrm{kg}$ ) or 3 multiple doses $(5 \mathrm{mg} / \mathrm{kg}$ ) of enrofloxacin subcutaneously every $24 \mathrm{~h}$ to healthy 6-mo-old Holstein steers demonstrated that active enrofloxacin drug concentrations in the ileum and colon exceeded plasma and interstitial fluid concentrations but were largely eliminated by $48 \mathrm{~h}$ after the last dose (Ferguson et al., 2018). In the Ferguson et al. (2018) study, E. coli counts in the feces were significantly decreased during peak drug concentrations; however, by $96 \mathrm{~h}$ after treatment, E. coli counts returned to baseline. Given that our sampling interval from the first to the second fecal sample was $2 \mathrm{~d}$, we may not have been able to characterize a decrease in E. coli enteric population in ENR calves during that interval. Furthermore, a potentially shorter effect of enrofloxacin on E. coli cfu per gram of feces, not following the Ferguson et al. (2018) findings of up to $96 \mathrm{~h}$ after treatment, could have been due to the significantly lower number of $E$. coli cfu per gram of feces in preweaned calves compared with postweaned calves. This can clearly be observed in our study when comparing E. coli cfu per gram counts from samples collected at $112 \mathrm{~d}$ with any other prior sampling time point (Figure 1).

In our study, tulathromycin served as an alternative to enrofloxacin for treatment of calves at high risk of BRD. Unexpectedly, we observed that calves in the TUL group had a significant increase in shedding of $E$. coli resistant to ciprofloxacin compared with CTL, although not to shedding levels as high as those observed in the ENR group (Figure 4). A possible explanation for this finding could be related to the co-selection of bacteria with fluoroquinolone resistance genes in the gastrointestinal tract of calves receiving TUL. This can be challenging to evaluate based solely on phenotypic approaches for evaluating antimicrobial susceptibility, due to the inherent resistance to macrolide drugs in Enterobacteriaceae (Barker, 1999). Nevertheless, E. coli has been proposed as a reservoir for macrolide resistance genes, and a study that evaluated the prevalence 
of acquired resistance to macrolides in commensal and clinical E. coli isolates, using PCR to detect resistant genes, identified multiple macrolide resistance genes, with the $m p h(\mathrm{~A})$ gene being the most common (Nguyen et al., 2009). Furthermore, a study conducted in children evaluated the effect of antimicrobial therapy on the quantification of plasmid-mediated quinolone resistance genes $q n r \mathrm{~A}, q n r \mathrm{~B}$, and $q n r \mathrm{~S}$, using real-time PCR in stool samples (Vien et al., 2012). The authors of that study demonstrated an increasing prevalence of $q n r \mathrm{~B}$ and an increased quantity of the $q n r \mathrm{~B}$ and $q n r \mathrm{~S}$ genes in the stools of children after $7 \mathrm{~d}$ of treatment with non-fluoroquinolone antimicrobial drugs, including macrolide drugs, and attributed fluoroquinoloneresistant bacteria selection process to co-selection. Our findings highlight the importance of considering potential adverse effects when using approaches based on antimicrobial drugs to control BRD in preweaned calves.

No significant difference in treatment effects of enrofloxacin or tulathromycin was observed on the shedding of E. coli resistant to ceftriaxone, a third-generation cephalosporin (Figure 5). A study by Pereira et al. (2014c) indicated that calves with a treatment history of receiving enrofloxacin, based on farm records, resulted in selection of isolates that presented phenotypic resistance not only to ciprofloxacin but also to ceftriaxone. Resistance to fluoroquinolones has previously been shown to co-select for class- 1 integrons and integronborn extended-spectrum $\beta$-lactam genes, because quinolone resistance genes (qnr) are situated in class-1 integron structures, which can also harbor extendedspectrum $\beta$-lactam resistance genes that would result in phenotypic resistance to third-generation cephalosporin drugs (Nordmann and Poirel, 2005; Wellington et al., 2013). The lack of phenotypic resistance to ceftriaxone observed in our study could have been due to the fact that calves in the study by Pereira et al. (2014c) may have received enrofloxacin for treatment of BRD, and not for metaphylaxis, therefore potentially altering the shedding pattern of resistant bacteria due to presence of disease. Another difference is that, in the study by Pereira and colleagues, Kirby-Bauer disk diffusion assays were used to evaluate susceptibility in only 3 fecal $E$. coli isolates per calf, not providing a quantitative assessment of shedding of resistant bacteria as our current study did. Our study used a quantitative approach, evaluating resistance in enteric $E$. coli, which delivers a profound improvement in the ability to detect differences between treatments and periods, simply due to the substantial increase in the number of isolates examined for resistance.

Although no significant effect of treatment group on ceftriaxone resistance was found, calves in all 3 groups had a high proportion of $E$. coli resistant to ceftriaxone up to $56 \mathrm{~d}$ after initial enrollment in the study (Figure 5). High proportions of E. coli nonsusceptible to third-generation cephalosporins among preweaned calves were also observed in one study that longitudinally collected weekly fecal samples for characterization of E. coli antimicrobial susceptibility (Pereira et al., 2014a). In that study, a high proportion $(>70 \%)$ of fecal $E$. coli nonsusceptible to ceftriaxone was observed up to $6 \mathrm{wk}$ of age in fecal samples of calves receiving saleable milk containing added antimicrobial drugs to simulate nonsaleable milk with residues (waste milk) fed to preweaned calves at dairy farms (Pereira et al., 2014b; Tempini et al., 2018). In the same study, control calves fed saleable milk with no drug residues also had a spike in the proportion of $E$. coli nonsusceptible to ceftriaxone at wk 1 of life $(>60 \%)$, but with a prompt decrease to less than $50 \%$ of fecal E. coli nonsusceptible to ceftriaxone by 3 wk of age, and less than $20 \%$ by 5 wk of age (Pereira et al., 2014a). In our study, calves of all groups were fed pooled pasteurized nonsaleable milk twice a day, and this could have affected the high pattern of shedding of $E$. coli resistant to other thirdgeneration antimicrobial drugs in nonsaleable milk. Nevertheless, this could also have influenced the evaluation of the effect of enrofloxacin and tulathromycin on selection of resistance to ceftriaxone due to a high proportion of $E$. coli present in the gut microbiota at enrollment. Therefore, treating calves with enrofloxacin when being fed saleable milk or milk replacer not containing drug residue could potentially result in a different longitudinal shedding pattern of $E$. coli resistant to third-generation cephalosporins.

For individual isolates selected for characterization using Kirby-Bauer sensitivity testing, only 2 resistance patterns were observed for all isolates with resistance to ciprofloxacin (Supplemental Table S3, https://doi.org/ 10.3168/jds.2019-17989). One important observation for one of these resistance patterns is that, in addition to being resistant to 11 of the 12 drugs tested, it included resistance to ceftriaxone, a third-generation cephalosporin. This is of great relevance because ceftriaxone and ciprofloxacin are the antimicrobial drugs of choice for treatment of important infections in humans caused by gram-negative bacteria, such as diarrhea caused by severe enterotoxigenic E. coli, and invasive Salmonella infections (Dennehy, 2019). Although ENR and TUL may have provided a selection advantage for the 2 multidrug resistance patterns carrying resistance to ciprofloxacin, this seems to dissipate given the new microbial environment. One explanation for this could be the fitness cost that has been previously observed for resistance genes, which results in a selective disadvantage (Hernando-Amado et al., 2017). 


\section{CONCLUSIONS}

We observed that treatment of calves at high risk of developing BRD with enrofloxacin resulted in a significantly higher proportion of shedding of ciprofloxacin-resistant $E$. coli in fecal samples for up to $28 \mathrm{~d}$ after treatment, compared with calves receiving metaphylactic treatment with tulathromycin or no treatment. Similarly, treatment of calves with tulathromycin resulted in a significantly higher proportion of ciprofloxacin-resistant E. coli in fecal samples for up to $7 \mathrm{~d}$ after treatment, compared with calves not receiving any metaphylactic drug treatment. These findings highlight the importance of considering potential selection of antimicrobial resistance when planning and selecting antimicrobials to be used for in calves at high risk of developing BRD.

\section{ACKNOWLEDGMENTS}

This work was supported by the USDA National Institute of Food and Agriculture (NIFA)/Hatch (Washington, DC), project accession \#1004072. Any opinions, findings, conclusions, or recommendations expressed in this publication are those of the authors and do not necessarily reflect the view of NIFA or the USDA. For her assistance in the project, the authors thank Debora Pedroso (Cornell University, Ithaca, NY). We also thank the farm for allowing our research team to conduct the study with their calves. The authors declare that there is no conflict of interest.

\section{REFERENCES}

Aidara-Kane, A. 2012. Containment of antimicrobial resistance due to use of antimicrobial agents in animals intended for food: WHO perspective. Rev. Sci. Tech. 31:277-287. https://doi.org/10.20506/ rst.31.1.2115.

Aldred, K. J., R. J. Kerns, and N. Osheroff. 2014. Mechanism of quinolone action and resistance. Biochemistry 53:1565-1574. https:// doi.org/10.1021/bi5000564.

Andersson, M. I., and A. P. MacGowan. 2003. Development of the quinolones. J. Antimicrob. Chemother. 51(Suppl. 1):1-11. https:/ /doi.org/10.1093/jac/dkg212.

Andraud, M., N. Rose, M. Laurentie, P. Sanders, A. Le Roux, R. Cariolet, C. Chauvin, and E. Jouy. 2011. Estimation of transmission parameters of a fluoroquinolone-resistant Escherichia coli strain between pigs in experimental conditions. Vet. Res. 42:44. https:// doi.org/10.1186/1297-9716-42-44.

Bailey, J. K., J. L. Pinyon, S. Anantham, and R. M. Hall. 2010. Commensal Escherichia coli of healthy humans: A reservoir for antibiotic-resistance determinants. J. Med. Microbiol. 59:1331-1339. https://doi.org/10.1099/jmm.0.022475-0.

Baptiste, K. E., and N. C. Kyvsgaard. 2017. Do antimicrobial mass medications work? A systematic review and meta-analysis of randomised clinical trials investigating antimicrobial prophylaxis or metaphylaxis against naturally occurring bovine respiratory disease. Pathog. Dis. 75:ftx083. https://doi.org/10.1093/femspd/ ftx083.
Barker, K. F. 1999. Antibiotic resistance: A current perspective. Br. J. Clin. Pharmacol. 48:109-124. https://doi.org/10.1046/j.1365-2125 .1999.00997.x.

Bywater, R. J. 2004. Veterinary use of antimicrobials and emergence of resistance in zoonotic and sentinel bacteria in the EU. J. Vet. Med. B Infect. Dis. Vet. Public Health 51:361-363. https://doi.org/10 .1111/j.1439-0450.2004.00791.x.

CLSI (Clinical and Laboratory Standards Institute). 2018. M100 Performance Standards for Antimicrobial Susceptibility Testing. 28th ed. Clinical and Laboratory Standards Institute, Wayne, PA.

Collignon, P. J., J. M. Conly, A. Andremont, S. A. McEwen, A. Aidara-Kane, and World Health Organization Advisory Group, Bogotá Meeting on Integrated Surveillance of Antimicrobial Resistance (WHO-AGISAR). 2016. World Health Organization ranking of antimicrobials according to their importance in human medicine: A critical step for developing risk management strategies to control antimicrobial resistance from food animal production. Clin. Infect. Dis. 63:1087-1093. https://doi.org/10.1093/cid/ciw475.

Constable, P. D. 2004. Antimicrobial use in the treatment of calf diarrhea. J. Vet. Intern. Med. 18:8-17.

Crosby, S., B. Credille, S. Giguere, and R. Berghaus. 2018. Comparative efficacy of enrofloxacin to that of tulathromycin for the control of bovine respiratory disease and prevalence of antimicrobial resistance in Mannheimia haemolytica in calves at high risk of developing bovine respiratory disease. J. Anim. Sci. 96:1259-1267. https:/ /doi.org/10.1093/jas/sky054.

Cummings, K. J., V. A. Aprea, and C. Altier. 2014. Antimicrobial resistance trends among Escherichia coli isolates obtained from dairy cattle in the Northeastern United States, 2004-2011. Foodborne Pathog. Dis. 11:61-67. https://doi.org/10.1089/fpd.2013.1605.

Dennehy, P. H. 2019. Infectious gastroenteritis. Introduction to Clinical Infectious Disease: A Problem-Based Approach. 1st ed. Springer, Syracuse, NY.

Dunlop, R. H., S. A. McEwen, A. H. Meek, R. C. Clarke, R. M. Friendship, W. D. Black, and A. N. Sharpe. 1998. Measurement of antimicrobial-resistant Escherichia coli in pig feces with a hydrophobic grid membrane filter interpreter system. Appl. Environ. Microbiol. 64:366-369. https://doi.org/10.1128/AEM.64.1.366-369.1998.

Fantin, B., X. Duval, L. Massias, L. Alavoine, F. Chau, S. Retout, A. Andremont, and F. Mentre. 2009. Ciprofloxacin dosage and emergence of resistance in human commensal bacteria. J. Infect. Dis 200:390-398. https://doi.org/10.1086/600122.

FDA. 2005. NADA 141-244. Freedom of Information summary: Original new animal drug application. DRAXXIN injectable solution (tulathromycin). For the treatment of bovine respiratory disease (BRD) associated with Mannheimia haemolytica, Pasteurella multocida, and Histophilus somni (Haemophilus somnus); for the control of respiratory disease in cattle at high risk of developing BRD associated with Mannheimia haemolytica, Pasteurella multocida, and Histophilus somni; and for the treatment of swine respiratory disease (SRD) associated with Actinobacillus pleuropneumoniae, Pasteurella multocida, Bordetella bronchiseptica, and Haemophilus parasuis. Accessed Aug. 12, 2020. https://animaldrugsatfda.fda .gov/adafda/app/search/public/document/downloadFoi/789.

FDA. 2008a. NADA 141-068. Freedom of Information summary: Supplemental new animal drug application. BAYTRIL 100 enrofloxacin injectable solution, beef and non-lactating dairy cattle. For the control of bovine respiratory disease (BRD) in beef and nonlactating dairy cattle at high risk of developing bovine respiratory disease (BRD) associated with Mannheimia haemolytica, Pasteurella multocida, Histophilus somni, and Mycoplasma bovis. Supplemental new animal drug application. Accessed Aug. 12, 2020. https:// animaldrugsatfda.fda.gov/adafda/app/search/public/document/ downloadFoi/607.

FDA. 2008b. NADA 141-068. BAYTRIL 100 enrofloxacin injectable solution, beef and non-lactating dairy cattle. To provide for the use of enrofloxacin in female dairy cattle less than 20 months of age. Accessed Aug. 12, 2020. https://animaldrugsatfda.fda.gov/ adafda/app/search/public/document/downloadFoi/604. 
FDA. 2012. Safe levels for extralabel use of drugs in animals and drugs prohibited from extralabel use in animals. Food and Drug Administration, White Oak, MD.

Ferguson, K. M., M. E. Jacob, C. M. Theriot, B. J. Callahan, T. Prange, M. G. Papich, and D. M. Foster. 2018. Dosing regimen of enrofloxacin impacts intestinal pharmacokinetics and the fecal microbiota in steers. Front. Microbiol. 9:2190. https://doi.org/10 $.3389 /$ fmicb.2018.02190.

Foditsch, C., R. V. V. Pereira, J. D. Siler, C. Altier, and L. D. Warnick. 2019. Effects of treatment with enrofloxacin or tulathromycin on fecal microbiota composition and genetic function of dairy calves. PLoS One 14:e0219635. https://doi.org/10.1371/journal .pone.0219635.

FOI. 2005. NADA 141-244: DRAXXIN Injectable Solution (tulathromycin). Food and Drug Administration, Washington, DC.

Heinrichs, A. J., and K. E. Lesmeister. 2005. Rumen development in the dairy calf. Pages 53-65 in Calf and Heifer Rearing. P. C. Garnsworthy, ed. Nottingham Univ. Press, Nottingham, UK.

Heins, B. D., D. V. Nydam, A. R. Woolums, R. D. Berghaus, and M. W. Overton. 2014. Comparative efficacy of enrofloxacin and tulathromycin for treatment of preweaning respiratory disease in dairy heifers. J. Dairy Sci. 97:372-382. https://doi.org/10.3168/ jds.2013-6696.

Hernando-Amado, S., F. Sanz-Garcia, P. Blanco, and J. L. Martinez. 2017. Fitness costs associated with the acquisition of antibiotic resistance. Essays Biochem. 61:37-48. https://doi.org/10.1042/ EBC20160057.

Hopkins, K. L., R. H. Davies, and E. J. Threlfall. 2005. Mechanisms of quinolone resistance in Escherichia coli and Salmonella: Recent developments. Int. J. Antimicrob. Agents 25:358-373. https://doi .org/10.1016/j.ijantimicag.2005.02.006.

Ives, S. E., and J. T. Richeson. 2015. Use of antimicrobial metaphylaxis for the control of bovine respiratory disease in high-risk cattle. Vet. Clin. North Am. Food Anim. Pract. 31:341-350. https://doi .org/10.1016/j.cvfa.2015.05.008.

Jacoby, G. A., J. Strahilevitz, and D. C. Hooper. 2014. Plasmid-mediated quinolone resistance. Microbiol. Spectr. 2. https://doi.org/10 .1128/microbiolspec.PLAS-0006-2013.

Jordan, D., S. G. Morris, P. Gill, L. M. Andersen, A. Chowdhury, A. E. Stevenson, and S. A. Spence. 2005. Mass screening for antimicrobial resistant Escherichia coli in dairy cows in northern New South Wales. Aust. Vet. J. 83:688-694. https://doi.org/10.1111/j .1751-0813.2005.tb13051.x

Mann, S., J. D. Siler, D. Jordan, and L. D. Warnick. 2011. Antimicrobial susceptibility of fecal Escherichia coli isolates in dairy cows following systemic treatment with ceftiofur or penicillin. Foodborne Pathog. Dis. 8:861-867. https://doi.org/10.1089/fpd.2010 .0751 .

Mesa, R. J., V. Blanc, A. R. Blanch, P. Cortes, J. J. Gonzalez, S. Lavilla, E. Miro, M. Muniesa, M. Saco, M. T. Tortola, B. Mirelis, P. Coll, M. Llagostera, G. Prats, and F. Navarro. 2006. Extendedspectrum beta-lactamase-producing Enterobacteriaceae in different environments (humans, food, animal farms and sewage). J. Antimicrob. Chemother. 58:211-215. https://doi.org/10.1093/jac/ dkl211.

Nguyen, M. C. P., P. L. Woerther, M. Bouvet, A. Andremont, R. Leclercq, and A. Canu. 2009. Escherichia coli as reservoir for macrolide resistance genes. Emerg. Infect. Dis. 15:1648-1650. https:// doi.org/10.3201/eid1510.090696.

Nordmann, P., and L. Poirel. 2005. Emergence of plasmid-mediated resistance to quinolones in Enterobacteriaceae. J. Antimicrob. Chemother. 56:463-469. https://doi.org/10.1093/jac/dki245.

O'Connor, A. M., C. Yuan, J. N. Cullen, J. F. Coetzee, N. da Silva, and C. Wang. 2016. A mixed treatment meta-analysis of antibiotic treatment options for bovine respiratory disease - An update. Prev. Vet. Med. 132:130-139. https://doi.org/10.1016/j.prevetmed 2016.07.003

Oliver, S. P., S. E. Murinda, and B. M. Jayarao. 2011. Impact of antibiotic use in adult dairy cows on antimicrobial resistance of veterinary and human pathogens: A comprehensive review. Foodborne Pathog. Dis. 8:337-355. https://doi.org/10.1089/fpd.2010.0730.
Pereira, R. V., S. Lima, J. D. Siler, C. Foditsch, L. D. Warnick, and R. C. Bicalho. 2016. Ingestion of milk containing very low concentration of antimicrobials: Longitudinal effect on fecal microbiota composition in preweaned calves. PLoS One 11:e0147525. https:// doi.org/10.1371/journal.pone.0147525.

Pereira, R. V., J. D. Siler, R. C. Bicalho, and L. D. Warnick. 2014a. In vivo selection of resistant $E$. coli after ingestion of milk with added drug residues. PLoS One 9:e115223. https://doi.org/10.1371/ journal.pone.0115223.

Pereira, R. V., J. D. Siler, R. C. Bicalho, and L. D. Warnick. 2014b. Multiresidue screening of milk withheld for sale at dairy farms in central New York State. J. Dairy Sci. 97:1513-1519. https://doi .org/10.3168/jds.2013-7421.

Pereira, R. V., J. D. Siler, J. C. Ng, M. A. Davis, Y. T. Grohn, and L. D. Warnick. 2014c. Effect of on-farm use of antimicrobial drugs on resistance in fecal Escherichia coli of preweaned dairy calves. J. Dairy Sci. 97:7644-7654. https://doi.org/10.3168/jds.2014-8521.

Rybak, M. J. 2006. Pharmacodynamics: Relation to antimicrobial resistance. Am. J. Infect. Control 34(Suppl. 1):S38-S45. https://doi .org/10.1016/j.ajic.2006.05.227.

Sims, D., I. Sudbery, N. E. Ilott, A. Heger, and C. P. Ponting. 2014. Sequencing depth and coverage: Key considerations in genomic analyses. Nat. Rev. Genet. 15:121-132. https://doi.org/10.1038/ $\operatorname{nrg} 3642$.

Smith, A. B., D. G. Renter, N. Cernicchiaro, X. Shi, J. S. Nickell, D. J. Keil, and T. G. Nagaraja. 2017. A randomized trial to assess the effect of fluoroquinolone metaphylaxis on the fecal prevalence and quinolone susceptibilities of Salmonella and Campylobacter in feedlot cattle. Foodborne Pathog. Dis. 14:600-607.

Spellberg, B., M. Blaser, R. J. Guidos, H. W. Boucher, J. S. Bradley, B. I. Eisenstein, D. Gerding, R. Lynfield, L. B. Reller, J. Rex, D. Schwartz, E. Septimus, F. C. Tenover, and D. N. Gilbert. 2011. Combating antimicrobial resistance: Policy recommendations to save lives. Clin. Infect. Dis. 52(Suppl. 5):S397-S428.

Tempini, P. N., S. S. Aly, B. M. Karle, and R. V. Pereira. 2018. Multidrug residues and antimicrobial resistance patterns in waste milk from dairy farms in Central California. J. Dairy Sci. 101:81108122. https://doi.org/10.3168/jds.2018-14398.

TerHune, T. N., T. L. Skogerboe, V. K. Shostrom, and D. J. Weigel. 2005. Comparison of pharmacokinetics of danofloxacin and enrofloxacin in calves challenged with Mannheimia haemolytica. Am. J. Vet. Res. 66:342-349. https://doi.org/10.2460/ajvr.2005.66.342.

Vaidya, V. K. 2011. Horizontal transfer of antimicrobial resistance by extended-spectrum $\beta$ lactamase-producing Enterobacteriaceae. J. Lab. Physicians 3:37-42. https://doi.org/10.4103/0974-2727 .78563

Vien, L. T. M., N. N. Q. Minh, T. C. Thuong, H. D. Khuong, T. V. T. Nga, C. Thompson, J. I. Campbell, M. de Jong, J. J. Farrar, C. Schultsz, H. R. van Doorn, and S. Baker. 2012. The co-selection of fluoroquinolone resistance genes in the gut flora of Vietnamese children. PLoS One 7:e42919. https://doi.org/10.1371/journal .pone.0042919.

Wellington, E. M., A. B. Boxall, P. Cross, E. J. Feil, W. H. Gaze, P. M. Hawkey, A. S. Johnson-Rollings, D. L. Jones, N. M. Lee, W. Otten, C. M. Thomas, and A. P. Williams. 2013. The role of the natural environment in the emergence of antibiotic resistance in gram-negative bacteria. Lancet Infect. Dis. 13:155-165. https:// doi.org/10.1016/S1473-3099(12)70317-1.

\section{ORCIDS}

R. V. Pereira (ํ) https://orcid.org/0000-0003-2028-8761

C. Altier (ㄴ) https://orcid.org/0000-0002-3740-9163

S. Mann ๑ https://orcid.org/0000-0003-1806-1154

D. Jordan $\odot$ https://orcid.org/0000-0002-1763-9551

L. D. Warnick @ https://orcid.org/0000-0001-9038-9176 